\title{
A Review on Inter-Satellite Optical Wireless Communication
}

\author{
Abhishek Sharma \\ Assistant Professor \\ Sri Sai University \\ Palampur, India
}

\author{
Vishal Kumar \\ Research Scholar \\ Sri Sai University \\ Palampur, India
}

\author{
Vipin Gupta \\ Associate Professor \\ Sri Sai College of Engg \& Tech \\ Badhani, Pathankot, India
}

\begin{abstract}
Inter-satellite optical wireless Communication has been emerged as a revolutionary technology in the field of communication. Since its induction in early 60 's, Is-OWC has revolutionized the communication industry with high sapped data transmission as well as high end security. In this paper, study about various aspects of Is-OWC as well as its advantages and applications. Also various modulation formats and their impacts in enhancing capacity of the system are discussed.
\end{abstract}

\section{Keywords}

Inter-Satellite Optical Wireless Link (Is-OWC), Radio Frequancy (RF), Radio over Fiber (RoF)

\section{INTRODUCTION}

Recent years has witnessed massive growth in the field of information and communication technology. All of this becomes possible with advancement and use of Light wave technology in the field of communication. With high speed, data transmission over larger distance has become possible [1]. This gave rise to the optical wireless communication (OWC) being applied commercially. Due to many advantages over tradition radio frequency signal (RF), OWC has been currently applied in number of applications. LASER has been used intensively due to their coherent properties and thus the same is used to transmit data in space to communicate between satellites orbiting in same or different orbits. This technology has been named as inter-satellite optical wireless communication (Is-OWC) [2]. With properties like high speed and high capacity, Is-OWC systems enable more effective, efficient and consistent operation of satellite system in years to come [3]. Thus by use of Is-OWC, an evolutionary space network can be formed from satellites which can serve as both backbone as well as user access nodes [4-36].

\section{Is-OWC SYSTEM}

The Figure 1.1 shows the optical communication between two satellites. Figure 1.2 shows how the inter-satellite laser links covers a large area of the Earth's surface.

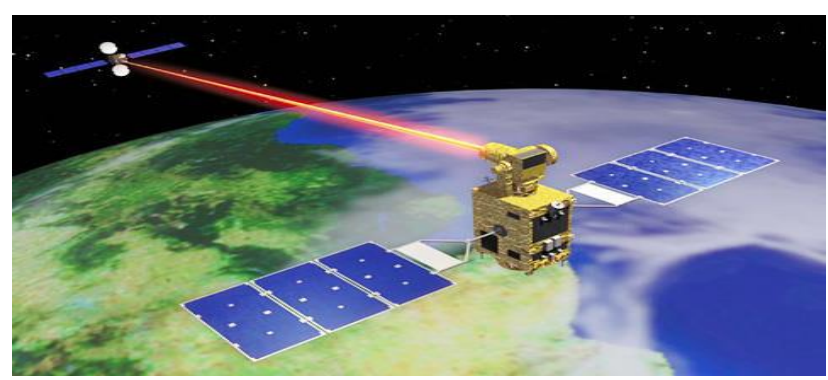

.Figure 1 Inter-satellite Optical communications [37]

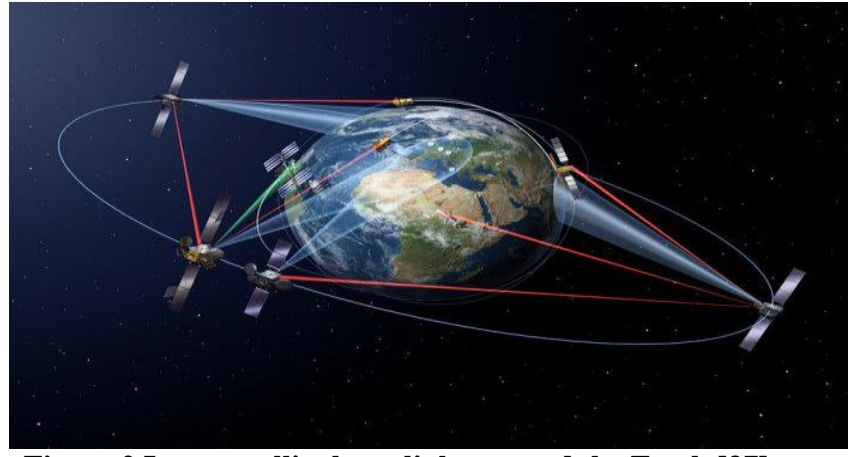

Figure 2 Inter-satellite laser links around the Earth [37]

Signal is being transmitted using free space optics between two satellites in Is-OWC [38]. The Figure 1.3 represents the basic block diagram of Inter-satellite Wireless Optical Communication (Is-OWC).

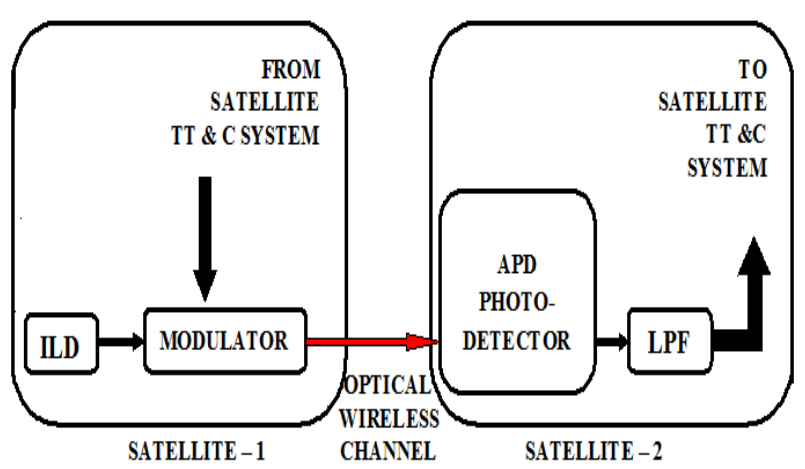

Figure 3 Is-OWC basic system block diagram for simplex communication [37]

\subsection{Transmitter}

The transmitting satellite consists of LASER which is used a source for generating light, satellite telemetry, tracking and communication system and an optical modulator. The IsOWC transmitter receives information from the satellite's Telemetry, Tracking and Communication (TT\&C) system [39]. The information which is generally transmitted by a satellite is same as the location of the satellite and tracking of attitude, picture capturing the data that is usually transmitted by a satellite are such as the satellite location and attitude tracking for isolated detecting satellite and speech data intended for handset system conveying satellite. The most substantial component in the system is light source as communication is done by transmitting light. Two types of optical light sources are used in optical communication, i.e., Light-emitting diode (LED) and injected laser diode (ILD). Formation of these devices is done by semiconductor 
materials with the interaction of positive and negative charges semiconductor yielding photons or light energy. Emission of light from ILD monochromatic, coherent and has inordinate vivacity making it appropriate for covering large distance for transmitting in free space [40-41].

\subsection{Propagation Medium}

Considering the circumstance of Is-OWC system, optical wireless channel is regarded as the transmission medium. OWC channel is vacuum which is free from losses like atmospheric losses contrary to free space optics which is introduced to several losses owing to weather and atmospheric attenuation [42-45]. Taking the ideal case, transmitting distance is the sole cause signal attenuation. At the transmitter and the receiver side, use of optical antennas or optical lenses is made. The optical antenna permits wider divergence of light beam recognition.

\subsection{Receiver}

The signal on the receiver side of the Is-OWC includes a photodiode and a low pass filter. The detection of the received signal is done by a photodiode which converts it into an electrical signal [46]. Photodiode comprises of positive and negative charged semiconductor connection, linked in reverse bias just like in an optical light source [47-88]. An electrical signal is generated when photons strike the junction. APD is used because of its distinctive feature of having very large amplification of low or weak light signals in free space optical transmission of data [49]. Amplification or avalanche occurrence in APD photo detector take place during introduction of charged electrons in excessive high electric field region and strike with neutral semiconductor atoms, hence creating additional carriers in huge quantity. PIN Diode can also be used in place of APD.

\section{RELATED WORK}

Many researchers have investigated the Is-OWC field in the recent years. Few of them are listed as:

Tianya Song et al. (2017) [50] studied the consequence of pointing errors on the average bit error probability (ABEP) of an inter-satellite laser communication link. The expression in form of closed-form in terms of the Marcum Q -function is computed for instant channel gain. This expression delivers excessive potential in additional presentation investigation and system optimization. Additional simplifications of these limits are achieved and invertible ABEP expressions are assumed. It is easy to gain diversity by using the invertible ABEP expressions linking to the ratio of the correspondent beam radius to the pointing error displacement standard jitter at the receiver.

Xiaohua Jia et al. (2017) [51] presented a satellite system attracting a huge amount of consideration from educational and industrial in past few years. Numerous satellites are launched which are used in predicting the weather, monitoring the environment and target surveillance. Downloading the data is one of the significant tasks which are composed in space to the ground servers via earth stations (ESs). Satellites move at great speed along their own orbits having restricted contact time with ESs therefore they may not be capable of downloading all the data they have to the ground on time. A cooperative system that permits satellites to divest data amongst themselves by means of inter-satellite links (ISLs) before interacting with the ES so that satellites carries the accurate quantity of data in accordance to the span of their interaction time with the ES and the output of downloading the data at the ES is maximized. An iterative optimization procedure is formed that mutually schedules data divest amid the satellites and downloading the data from satellites to the ES. Many simulations have been carried out for estimating the efficiency of the projected technique. The results prove that the data downloading throughput is increased considerably by using ISL. Throughput can even reach close to $100 \%$ of the size of the ES.

Hemani Kaushal et.al (2017) [52] discussed that free space optical (FSO) communication improved drastically because of its exceptional features: greater bandwidth, spectrum free from license, great amount of data, rapid deploy ability, fewer power and mass necessities. In spite of inordinate potential of FSO communication, restriction in working is there due adversative effects (viz., absorption, scattering, and turbulence) of the atmospheric channel. Atmospheric turbulence is a huge challenge among the three leading to severe deprivation in the performance of bit error rate making the communication link viable. Various problems are faced by FSO communication system for ground-to-satellite/satelliteto-ground and inter-satellite links. For high link accessibility and consistency numerous performance mitigation methods are represented. A major problem is posed to the working of the system due to much impairment for ground-tosatellite/satellite-to-ground and inter-satellite links. For combating the adversative effects of environment numerous methods for physical and other layers is provided. It also exclusively represents a newly established method which used orbital angular momentum for operating the high capacity benefit of optical carrier in case of space-based and near-Earth optical communication links. Facts on using optical backhaul links based on space for providing high volume and lower value backhaul solutions.

Pablo G. Madoery et al. (2016) [53] examined that the Earth surveillance satellite constellations are ascending as a new prototype having significant benefits in contrast with traditional systems. The perception of Segmented Architecture is for transforming these clutters into a dispersed and associated arrangement for exploiting resource sharing. Numerous technical encounters are executed like packetized space networking communications. Because of the restricted accessibility of transmission of data, these can be demonstrated as delay and disruption tolerant networks (DTN). The author proposed a specific case study of numerous flight-formation satellites envisioned for the reflection of the Earth. Both the accessibility of Earth-tospace links and their obliging combination of inter-satellite links are taken into consideration. Diverse routing schemes such as Contact Graph Routing (CGR) and its extension Multi-Graph CGR (MG-CGR) are examined and matched.

Radhika Radhakrishnan et al. (2016) [54] states that, an entire new session of navigation tasks, communications, remote sensing is aided by small satellite systems. They also enable research in scientific era for civilian and military purpose both. Small satellites are useful by forming clusters in many scientific missions like mapping of gravity, keeping tracks of forest fires, discovering water resources, etc. they are helpful in many ways. This enables an improved understanding of the environment of Earth and effectively providing an economical admittance of multi-satellite solution. Better spatial and temporal resolution of the aim is provided by these clusters. Upcoming space undertakings are intended to turn into high complexity and work at a large distance from Earth supporting numerous operations with minimum human interactions. As the level of autonomy is growing, it has become a stage for isolated communication networks for 
providing communication among spacecraft. They will need a way to organize and preserve vibrant routes, accomplish intermediate nodes, and reconfigure itself for attaining objectives of missions. Therefore, inter-satellite communication is an important feature once satellites hover in formation. The implementation is based the open system interconnection (OSI) model. The design parameters are valid to the three layers of the OSI model, i.e., physical, data link, and network layer. Comprehensive framework also represents useful variables for attaining satellite communications for numerous minor satellite tasks.

\section{APPLICATIONS OF Is-OWC}

Light wave system is utilized in numerous forms associated with satellite communication. The variety of uses of Intersatellite Wireless Optical Communication (Is-OWC) can be generally subdivided as:

- Broadband/Internet links

- Deep Space Optical Communications

- $\quad$ Satellite launch support

The Is-OWC has other application areas also that include satellite aircraft, deep space, under water and terrestrial communications [55-57].

\section{ADVANTAGES OF Is-OWC}

The conventional ways of wireless communication were completed by Radio Frequency (RF) or Microwave links. In the past years, the inter-satellite optical wireless communication has become famous become due to the following benefits:

1) High Data Rates: It is possible to improve fast intersatellite links with the help of light as carrier frequency [58]. Data rates up to numerous Gbps are effortlessly accomplished in optical inter-satellite communication.

2) Unlicensed Spectrum: In optical inter-satellite communication requirement of license of spectrum or frequency coordination is not needed for other users as in radio and microwave systems. Interference is not a chief concern from or to systems and also, point-to-point laser signal is tremendously hard to interrupt, making it very secure and safe.

3) Smaller Wavelength: The wavelength of light is about 1000 times smaller than microwaves. Hence, there is a larger difference in the signal wavelength of optical inter-satellite communication over RF/Microwave communication system. In other words, the RF/Microwave wavelength is much longer as compared to LASER light wavelength. Therefore, variation from RF/Microwave to light waves signifies the decrease of the beam width of the signal of the order of magnitude [59]. Intensity on the receiver side is increased due to the reduced beam width and crosstalk between closer operating links is also reduced.

4) Huge Bandwidth: In a particular communication system, the amount of data which is transported is directly related with modulated carrier bandwidth. Bandwidth of about $2000 \mathrm{THz}$ can be used by by means of optical carrier of high frequencies. Hence, assured increase in capacity of information is offered by optical inter-satellite communication in comparison to communications systems that are based on radio frequency. The reason behind this being, optical carrier frequency (including infrared, visible and ultra violet frequencies) much superior on the electromagnetic spectrum than radio/microwave frequency. The bandwidth of the frequency that is functional in RF/Microwave range is moderately low.

5) Reducing Antenna Size: The size requirement of the transmitter and receiver antenna is very large and also bulky if $\mathrm{RF} / \mathrm{Microwave}$ system is to be active. But in optical intersatellite communication, the antenna size gets reduced to centimeters only, as its carrier frequency is very large, therefore, decreasing the heaviness of the satellite and also reducing power requirement for the communication system [60-61]. The cost of the satellite is also greatly reduced by decreasing the size and height of payloads of the satellite.

6) Narrow Beam Size: The optical radiation is recognized for its particularly narrow beam and a typical laser beam has a diffraction restricted divergence between 0.01 to $0.1 \mu \mathrm{rad}$. This demonstrates that transmitting power is focused with a narrow range giving an optical inter-satellite link providing ample isolation from its probable interferers. It is also very tough jamming or tapping the signal with a narrow beam angle ensuring more safety.

7) The other advantages include immunity to the radio frequency interfaces; faster communication, power efficiency, high precision and above all wireless optical communication doesn't pollute the environment with electromagnetic radiations. All the details specified are dynamic in an optical inter-satellite communication system since it can decrease the payloads and therefore decreasing the cost.

\section{CONCLUSION}

As technology has evolved, means of communication have also changed. Is-OWC is a remarkable technology which has been widely used and explored for terrestrial communication and broadcast purpose. After studying the related work of this field it is concluded that there is need to do more work on the various quality factors like increase in bandwidth, to enhance the number of users that can participate in the communication simultaneously. Hence further enhancements can be done in order to remove these flaws form the communication system by using trendy mechanisms. Various advanced modulation formats can be used as well for enhancing existing capacity of the Is-OWC systems.

\section{REFERENCES}

[1] Someda SG. Electromagnetic Waves. Great Britain: Chapman \& Hall, 1998.

[2] Karafolas N et al. (2000). Optical satellite networks, Journal of Lightwave Technology, 18 (12), 1792-1806.

[3] Franck L et al. (2002). Routing in networks of intersatellite links, IEEE Transactions on Aerospace and Electronic System, 38 (3), 902-917.

[4] Sharma, A., Chaudhary, S., Thakur, D., Dhasratan, Vigneswaran "A Cost-Effective High-Speed Radio over Fibre System for Millimeter Wave Applications", Journal of Optical Communications, Published Online: 2017-12-15 | DOI: https://doi.org/10.1515/joc-2017-0166

[5] Sushank Chaudhary, Priyanka Chauhan, Abhishek Sharma, "High Speed $4 \times 2.5$ Gbps-5 GHz AMI-WDM-RoF Transmission System for WLANs", Journal of Optical Communications, Published Online: 2017-07-18 | DOI: https://doi.org/10.1515/joc-2017-0082

[6] Sushank Chaudhary, Deepika Thakur, Abhishek Sharma, "10 Gbps-60 GHz RoF Transmission System for $5 \mathrm{G}$ 
Applications", Journal of Optical Communications, Published Online: 2017-07-22 | DOI: https://doi.org/10.1515/joc-20170079

[7] Rudrakshi Kapoor, Abhishek Sharma, Sushank Chaudhary, "Empirical Evaluation of 4 QAM and 4 PSK in OFDM-based Inter-Satellite Communication System", Journal of Optical Communications, Published Online: 2017-06-29 | DOI: https://doi.org/10.1515/joc-2017-0059

[8] Abhishek Sharma, Neha Chaudhary, Sushank Chaudhary, "6 $\times 20$ Gbps Hybrid WDM-PI Inter-satellite System under the Influence of Transmitting Pointing Errors", Journal of Optical Communications, Published Online: 2016-05-27 | DOI: https://doi.org/10.1515/joc-2015-0099

[8] Abhishek Sharma, Neetu, "ANALYSIS AND MITIGATION OF RECEIVER POINTING ERROR ANGLE ON INTER-SATELLITE COMMUNICATION" International Journal of Innovative Technology and Research, Volume 3, Issue 6, Pages 2540-2544, Nov 2015

[9] Sushank Chaudhary, Abhishek Sharma, Neetu, "6 x 20Gbps Long Reach WDM-PI based High Altitude Platform Inter-Satellite Communication System" International Journal of Computer Applications, Volume 122, Issue 22, Pages 4145, July 2015

[10] Savita Rana Abhishek Sharma, "Comprehensive study of Radio over Fiber with different Modulation Techniques - A Review", International Journal of Computer Applications, Volume 170, Issue 4, Pages 22-25, Aug 2017 DOI: 10.5120/ijca2017914829

[11] Abhishek Sharma, Deepika Thakur, "A Review on WLANs with Radio-Over-Fiber Technology", International Journal of Electronics and Communication Engineering (IJECE), Volume 6, Issue 5, Pages 1-6, Aug 2017.

[12] Kanika Thakur Abhishek Sharma, "Comparison of MDRZ, CSRZ and DRZ schemes using different Communiation Channels", International Journal of Computer Applications, Aug 2017 DOI: 10.5120/ijca2017915106

[13] Kanika Thakur Abhishek Sharma, "Study of Radio over Fiber with Different coding Channel - A Review", International Journal of Computer Applications, Aug 2017, DOI: 10.5120/ijca2017915033

[14] Abhishek Sharma, Priyanka Chauhan, "A Study of Radio over Fiber Technology in WLAN Applications" International Journal for Research in Applied Science \& Engineering Technology (IJRASET) Volume 5 Issue VIII, Page 416-420, August 2017

[15] Abhishek Sharma, Rudrakshi Kapoor, "STUDY OF VARIOUS CHALLENGES IN Is OWC: A Review", International Journal for Research in Applied Science \& Engineering Technology (IJRASET) Volume 5 Issue VIII, Page 802-807, August 2017

[16] Abhishek Sharma, Savita Rana, "Implementation of Radio over Fiber Technology with different filtration techniques" International Journal for Research in Applied Science \& Engineering Technology (IJRASET) Volume 5 Issue VIII, Page 783-789, August 2017.

[17] A. Amphawan and S. Chaudhary, "Free-space optical mode division multiplexing for switching between millimeterwave picocells," in International Conference on Optical and Photonic Engineering (icOPEN2015), 2015, pp. 95242H95242H-6.
[18] A. Amphawan, S. Chaudhary, and V. Chan, "2 x 20 Gbps-40 GHz OFDM Ro-FSO transmission with mode division multiplexing," Journal of the European Optical Society-Rapid publications, vol. 9, 2014.

[19] A. Amphawan, S. Chaudhary, R. Din, and M. N. Omar, "5Gbps $\mathrm{HG} 0,1$ and $\mathrm{HG} 0,3$ optical mode division multiplexing for RoFSO," in Signal Processing \& Its Applications (CSPA), 2015 IEEE 11th International Colloquium on, 2015, pp. 145-149.

[20] A. Amphawan, S. Chaudhary, T. Elfouly, and K. Abualsaud, "Optical mode division multiplexing for secure Ro-FSO WLANs," Advanced Science Letters, vol. 21, pp. 3046-3049, 2015.

[21] U. Bansal, K. Kaur, and S. Chaudhary, "Role of laser linewidth in high speed DWDM system by incorporating duobinary modulation scheme," International Journal of Computer Applications, vol. 109, 2015.

[22] S. Chaudhary and A. Amphawan, "The role and challenges of free-space optical systems," Journal of Optical Communications, vol. 35, pp. 327-334, 2014.

[23] S. Chaudhary and A. Amphawan, "High-speed millimeter communication through radio-over-free-space-optics network by mode-division multiplexing," Optical Engineering, vol. 56, p. 116112, 2017.

[24] S. Chaudhary, A. Amphawan, and K. Nisar, "Realization of free space optics with OFDM under atmospheric turbulence," Optik-International Journal for Light and Electron Optics, vol. 125, pp. 5196-5198, 2014.

[25] S. Chaudhary, P. Bansal, and M. Lumb, "Effect of beam divergence on WDM-FSO transmission system," International Journal of Computer Applications, vol. 93, 2014.

[26] S. Chaudhary, P. Bansal, and G. Singh, "Implementation of FSO network under the Impact of atmospheric turbulences," International Journal of Computer Applications, vol. 75, 2013.

[28] S. Chaudhary, N. Chaudhary, S. Sharma, and B. Choudhary, "High Speed Inter-Satellite Communication System by Incorporating Hybrid Polarization-Wavelength Division Multiplexing Scheme," Journal of Optical Communications.

[29] S. Chaudhary and S. Sharma, "Role of turbulences in WDM-polarization interleaving scheme based inter-satellite communication system," International Journal of Computer Applications, vol. 104, 2014

[30] P. Kaur, R. Kaur, and S. Chaudhary, "Implementation of High Speed Long Reach Hybrid Radio over Multimode Transmission System," International Journal of Computer Applications, vol. 91, 2014.

[31] R. Kaur and S. Chaudhary, "Simulative investigation of laser line-width and channel spacing for realization of DWDM systems under the impact of four wave mixing," Journal of Optical Communications, vol. 35, pp. 157-165, 2014.

[32] V. Sharma, "High speed CO-OFDM-FSO transmission system," Optik-International Journal for Light and Electron Optics, vol. 125, pp. 1761-1763, 2014.

[33] V. Sharma and S. Chaudhary, "Implementation of hybrid OFDM-FSO transmission system," International Journal of Computer Applications, vol. 58, 2012. 
[34] V. Sharma and S. Kumar, "Empirical evaluation of wired-and wireless-hybrid OFDM-OSSB-RoF transmission system," Optik-International Journal for Light and Electron Optics, vol. 124, pp. 4529-4532, 2013.

[35] A. Amphawan, S. Chaudhary, and T.-K. Neo, "HermiteGaussian mode division multiplexing for free-space optical interconnects," Advanced Science Letters, vol. 21, pp. 30503053, 2015.

[36] K. K. Upadhyay, S. Srivastava, N. Shukla, and S. Chaudhary, "High-Speed 120 Gbps AMI-WDM-PDM Free Space Optical Transmission System," Journal of Optical Communications.

[37] Shekhar S and Gupta A., "Inter-Satellite Optical Wireless Communication Link (IsOWC)", LAP Lambert Academic Publishing, 2014.

[38] Chan V.W.S, "Free space optical communication", Journal of Lightwave Technology, 24 (12), 4750-4762.

[39] Moli-Sanchez L et al., "Performance analysis of quantum cryptography protocols in optical earth-satellite and intersatellite links", IEEE Journal on Selected Areas in Communications, 27 (9), 1582-1590.

[40] Dang A, "Simultaneous Acquisition and Track Scheme with Multiple Terminals Based on Subspace Method for Optical Satellite Networks", IEEE Transactions on Aerospace and Electronic Systems, 46 (1), 263-277.

[41] Ghosna F.J. et al., "Pulse Position Modulation Coding Schemes for Optical Inter-satellite Links", Journal of IET, 46 (4), 290-291.

[42] Donner A et al., "Satellite networks for aeronautical communication: traffic modeling and link load analysis", IET Communications, 4 (13), 1594-1606.

[43] Sodnik Z, et al., "Optical Inter-satellite Communication", IEEE Journal of Selected Topics in Quantum Electronics, 16 (5), 1051-1057.

[44] Liu X, "Source-induced fading of a multiple-inputsingle-output optical satellite communication system described by beta functions", IET Optoelectronics, 5 (1), 2835 .

[45] Liu X, "Optimization of satellite optical transmission with correlated sways", IET Communications, 5 (8), 11071112 .

[46] Chatzidiamantis ND, “Adaptive Subcarrier PSK Intensity Modulation in Free Space Optical System", IEEE Transactions on Communications, 59 (5), 1368-1377.

[47] Xie W et al., "Received Signal Strength Reduction Analysis Based on the Wavelet Model in Inter-satellite Laser Communication", IEEE Journal of Lightwave Technology, 29 (15), 2327-2332
[48] Yang Y et al., "Research Bit Error Rate in the Presence of Local Wave front Aberration in Inter-satellite Laser Communication", IEEE Journal of Lightwave Technology, 29 (19), 2893-2898.

[49] Arruego I et al., "In-orbit measurement of SET and DD effects on optical wireless links for intra-satellite data transmission", IEEE Transactions on Nuclear Science, 58 (6), 3067-3075.

[50] Song T et al., "Impact of Pointing Errors on the Error Performance of Inter-satellite Laser Communication", Journal of Lightwave Technology, 35 (14), 3082-3091.

[51] Jia X et al., "Collaborative Data Downloading by Using Inter-Satellite Links in LEO Satellite Networks", IEEE Transactions on Wireless Communications, 16 (3), 15231532.

[52] Kaushal H and Kaddum G, "Optical Communication in Space: Challenges and Mitigation Techniques", IEEE Communications, 19 (1), 57-96.

[53] Madoery PG et al., "Analysis of Communications Strategies for Earth Observation Satellite Constellations", IEEE Transactions, 14 (6), 2777-2782.

[54] Radhakrishnan $\mathrm{R}$ et al., "Survey of Inter-Satellite Communication for Small Satllite Systems: Physical Layer to Network Layer View", IEEE Communication, 18 (4), 24422473.

[55] Wang Y, Li R and Zhao R, "Research of Signal in Space Integrity Monitoring Based on Inter-Satellite Links", IEEE Journal of Electronics, 24 (2), 439-444.

[56] Tang Y, Wang Y and Chen J, "High-sensitive acquisition of signals for Inter-Satellite links of navigation constellation", Electronics Letters, 51 (23), 1879-1880.

[57] Aravanis AI et al., "Power Allocation in Mulitbeam Satellite Systems: A Two-Stage Multi-objective Optimization, IEEE Transactions on Wireless Communications", 14 (6), $3171-3182$

[58] Chen $\mathrm{H}$ et al., "Approach for earth observation satellite real-time and playback data transmission scheduling", Journal of Systems Engineering and Electronics, 26 (5), 982-992.

[59] Uysal M., "Optical Wireless Communications", Switzerland: Springer International Publishing, 2016.

[60] Sanctis MD et al., "Satellite Communications Supporting Internet of Remote Things", IEEE Internet of Things Journal, 3 (1), 113-123.

[61] Huang J et al., "Adaptive Modulation and Coding Techniques for Global Navigation Satellite System InterSatellite Communication Based On The Channel Condition", IET Communication, 10 (16), 2091-2095. 